IBD

\title{
Cathelicidin can reverse intestinal fibrosis in models of colitis
}

A recent study has shown that the antimicrobial peptide cathelicidin has antifibrogenic effects in both in vivo and in vitro models of colitis. This finding could have important implications for patients with IBD, particularly those with Crohn's disease and strictures (intestinal fibrosis).

"Despite aggressive anti-inflammatory treatment, there is no way to prevent or cure intestinal fibrosis," says Hon Wai Koon, corresponding author. "Therefore, new therapeutic solutions against intestinal fibrosis are actively sought after." Koon and his colleagues study the anti-inflammatory effects of cathelicidin (also known as LL-37 in humans and mCRAMP in mice) in intestinal inflammation. They were intrigued as to whether cathelicidin might also possess antifibrogenic properties, especially as this molecule has previously been shown to suppress collagen synthesis-an important fibrotic response.

The researchers used two mouse models of colitis in their investigation, both of which are associated with increased collagen expression: trinitrobenzene sulphonic acid (TNBS)-induced colitis and Salmonella-typhimurium-induced caecal inflammation. Administration of cathelicidin (via intracolonic enema or injection with lentiviral particles expressing the cathelicidin gene) in both of these mouse models was found to attenuate collagen synthesis and deposition. "In terms of cell culture, we used a cultured human CCD-18Co colonic fibroblast cell line and human primary intestinal fibroblasts," explains Koon. "Collagen expression was suppressed by cathelicidin."

The team found that the antifibrogenic property of cathelicidin is ERK dependent. They also add that, although the antifibrogenic effect of cathelicidin is novel, it complements the known anti-inflammatory effects of this peptide.

"We might consider administering cathelicidin to patients with strictures," says Koon. A cathelicidin-overexpressing

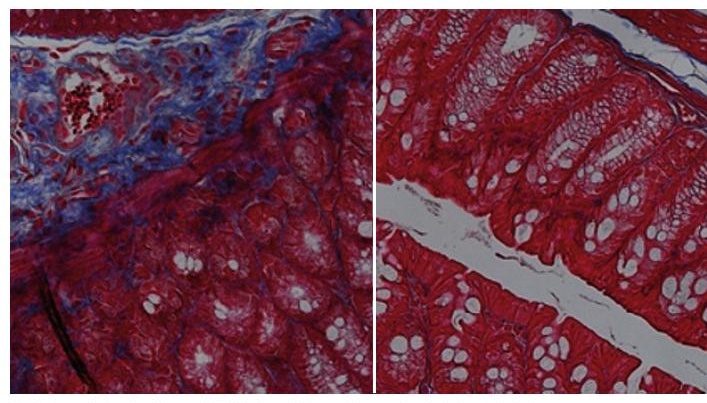

TNBS colitis (left) and TNBS colitis with cathelicidin (right). Collagen is stained blue. Courtesy of H. W. Koon

bacteria already exists that can be delivered to the gut. "Maybe we can use this bacterial strain to treat intestinal fibrosis," Koon adds. In addition, this study might point to potential antifibrogenic effects of other antimicrobial peptides.

Isobel Leake

Original article Yoo, J. H. et al. Anti-fibrogenic effects of the anti-microbial peptide cathelicidin in murine colitisassociated fibrosis. Cell. Mol. Gastroenterol. Hepatol. doi:10.1016/j.jcmgh.2014.08.001 